\title{
Trauma abdominal contuso por golpe con manubrio de bicicleta
}

\author{
Blunt abdominal trauma due to handlebar injury
}

\author{
María Jesús Irarrázaval Mainguyague a Josefina Sáez Binellia, Catalina Kychenthal Loyola ${ }^{a}$, \\ María Soledad Loyola Zunino ${ }^{b}$, Fernando Vuletin Solís ${ }^{a}$, Juan Carlos Pattillo Silva ${ }^{a}$
}

\author{
aSección de Cirugía Pediátrica, División de Cirugía, Escuela de Medicina, Facultad de Medicina, Pontificia Universidad Católica de Chile. \\ Santiago, Chile \\ bSección de Imagenología Intervencional, Departamento de Radiología, División de Imágenes, Laboratorios y Patologías, Escuela de Medicina, \\ Facultad de Medicina, Pontificia Universidad Católica de Chile. Santiago, Chile
}

Recibido: 18 de diciembre de 2019; Aceptado: 16 de marzo de 2020

¿Qué se sabe del tema que trata este estudio?

Los accidentes con lesiones por manubrio de bicicleta son una causa frecuente de trauma abdominal en niños. En Chile, no existen artículos que traten este tema.

\section{¿Qué aporta este estudio a lo ya conocido?}

En este trabajo alertamos acerca de los distintos mecanismos de trauma provocados por manubrio de bicicleta, haciendo énfasis en el manejo conservador del trauma abdominal contuso en pacientes pediátricos.

\section{Resumen}

Introducción: Los accidentes en bicicleta son una causa frecuente de trauma abdominal contuso en pediatría. En Chile no existen publicaciones científicas que traten sobre las lesiones abdominales por manubrio de bicicleta, su presentación y manejo. Objetivo: Describir tres casos clínicos de trauma abdominal contuso provocados por manubrio de bicicleta en niños, ilustrar las distintas lesiones observadas, sus formas de presentación y manejo. Casos Clínicos: 1) Paciente masculino, 11 años, consulta tras golpe con manubrio de bicicleta en epigastrio; en la Tomografía Computada (TC) de abdomen y pelvis se describió neumoretroperitoneo. Se realizó laparotomía exploradora, reparándose una perforación duodenal. 2) Paciente masculino, 14 años, consulta tras golpe en el hipocondrio izquierdo con el manubrio de la bicicleta; en la TC se evidenció fractura esplénica con múltiples laceraciones. Por la presencia de sangrado activo se trató con angioembolización, lográndose resolución de la lesión y viabilidad del órgano luego de 6 semanas de seguimiento. 3) Paciente masculino, 9 años, ingresó tras sufrir golpe con manubrio de bicicleta en el hipocondrio derecho. En TC se observó una laceración hepática, que fue manejada de forma expectante, con resolución de la lesión luego de 8 semanas de seguimiento. Todos los pacientes tuvieron una evolución favorable.
Palabras clave:

Trauma abdominal contuso;

bicicleta; niños 
Conclusión: El trauma abdominal contuso por golpe con manubrio de bicicleta puede ser potencialmente grave en pacientes pediátricos, pudiendo afectar órganos sólidos y vísceras huecas. El manejo no quirúrgico cada vez es más utilizado, logrando altas tasas de éxito en pacientes estables. Los pacientes inestables o en los que se sospeche perforación de víscera hueca, requerirán cirugía como primera aproximación.

\begin{abstract}
Introduction: Bicycle accidents are a frequent cause of blunt abdominal trauma in children. In Chile, there are no scientific articles about such accidents, their presentation and management. Objective: The aim of this study is to describe three cases of blunt abdominal trauma due to handlebar injury in children, in order to illustrate the different kinds of lesions, their presentation, and management. Clinical Cases: 1) 11-year-old boy presented to Emergency Department (ED) after falling on a bicycle handlebar, hitting his epigastric region. A CT scan showed signs of duodenal perforation. A laparotomy was performed and the duodenal perforation repaired. 2) 14-year-old boy seen at ED after a bicycle accident in which the handlebar hit him in the abdomen area. A CT scan showed a splenic injury with multiple lacerations and active bleeding that was treated with angioembolization. After 6 weeks of follow-up, he presented resolution of the lesion and viability of the spleen. 3) 9-year-old boy admitted due to a hit with the bicycle handlebar on the abdomen area. A CT scan showed a hepatic injury that was managed with non-surgical procedures, achieving resolution of the lesion after 8 weeks of follow-up. Conclusion: Blunt abdominal trauma caused by handlebar can be potentially serious in pediatric patients, since it may affect solid and hollow abdominal viscera. Non-surgical management is becoming more used for stable patients, achieving high success rates. Unstable patients or those with suspicion of hollow viscera perforation will require surgery as first approach.
\end{abstract}

Keywords:

Blunt abdominal trauma; bicycle; children

\section{Introducción}

El trauma es una causa importante de morbimortalidad en pediatría. En Chile, durante el año 2017 el $40,1 \%$ de las muertes entre los 1 y 19 años fueron secundarias a trauma ${ }^{1}$. Los accidentes en bicicleta dan cuenta importante del total de traumatismos en niños ${ }^{2-4}$. Según los registros de nuestro país, durante el año 2016, de todos los lesionados a causa de accidentes de tránsito en que hubo participación de ciclistas, que fueron 3.326 , el 14\% correspondió a menores de 18 años (489 lesionados) $)^{5}$. La prevención de lesiones graves asociadas a este tipo de accidentes ha estado centrada en el uso de casco, con lo que se ha logrado reducir la incidencia de lesiones cerebrales ${ }^{2,4}$. Sin embargo, las lesiones de órganos intraabdominales siguen siendo frecuentes ${ }^{6}$.

Los accidentes en bicicleta pueden causar daño por dos mecanismos: caída desde ella, asociada a traumatismo cráneo encefálico, o golpe con alguna de sus partes, más comúnmente con el manubrio ${ }^{7}$. Ante un golpe directo en la zona del abdomen, un paciente pediátrico corre mayor riesgo de sufrir lesiones de órganos internos en comparación con un paciente adulto ${ }^{2}$. En el cuerpo de un niño, las fuerzas son transmitidas con mayor facilidad a través de la pared abdominal y existe mayor superficie relativa de órganos sólidos como el bazo y el hígado ${ }^{2}$.
El tipo de lesiones causadas por estos accidentes puede ser diverso. En la región abdominal, la más frecuentemente afectada ${ }^{7,8}$, se describen lesiones de vísceras huecas y de órganos sólidos. Las lesiones de vísceras huecas pueden incluir perforación o hematoma a lo largo del tracto gastrointestinal y su mesenterio. Dentro de las lesiones de órganos sólidos, las más frecuentes son hepáticas, esplénicas, pancreáticas y rena$\mathrm{les}^{7}$; para graduar su magnitud se utiliza la escala creada por la Asociación Americana de Cirugía de Trauma (AAST, por su sigla en inglés) que está basada en una descripción anatómica y va de I a V, desde las lesiones menos graves a las más graves ${ }^{9}$.

Tradicionalmente, el hallazgo de líquido libre o lesión de órgano sólido ha sido tratado con cirugía de emergencia ${ }^{8}$. Sin embargo, la alta morbilidad asociada ha favorecido el manejo conservador, que ha demostrado tener mejores resultados a corto y largo plazo ${ }^{6}$. El uso de imágenes, como la tomografía computada (TC), ha permitido identificar la localización de las lesiones y contribuir a determinar la gravedad de éstas, entregando elementos para definir la mejor alternativa terapéutica.

No existen en nuestro país publicaciones científicas que traten acerca de este tipo de accidentes en población pediátrica, a pesar de su alta frecuencia y potencial gravedad. El objetivo de este manuscrito es describir tres casos clínicos de trauma abdominal contuso en ni- 
ños, provocados por manubrio de bicicleta, e ilustrar distintos tipos de lesiones posibles de observar, con sus formas de presentación y respectivo manejo.

\section{Casos Clínicos}

Los pacientes presentados a continuación fueron tratados siguiendo los protocolos del programa "Advanced Trauma Life Suport” (ATLS) del American College of Surgeons, se presentan los hallazgos positivos relevantes.

\section{Caso 1}

Paciente masculino, sano, de 11 años. Ingresó 90 minutos después de una caída en bicicleta con impacto de manubrio en zona epigástrica. Taquicárdico (104 lpm) e hipertenso (132/90) mmHg, con un Glasgow Coma Scale (GCS) de 15 puntos. Al examen físico se encontraba decaído, levemente deshidratado, bien perfundido, con dolor abdominal intenso y resistencia muscular a la palpación. Se constató una lesión erosiva en el epigastrio (figura 1).

Se realizó Tomografía Computada (TC) de abdomen y pelvis con contraste que informó neumoretroperitoneo (figura 2). Dado sospecha de lesión duodenal, se realizó una laparotomía exploradora, en la que se evidenció perforación de $2 \mathrm{~cm}$ entre la segunda y

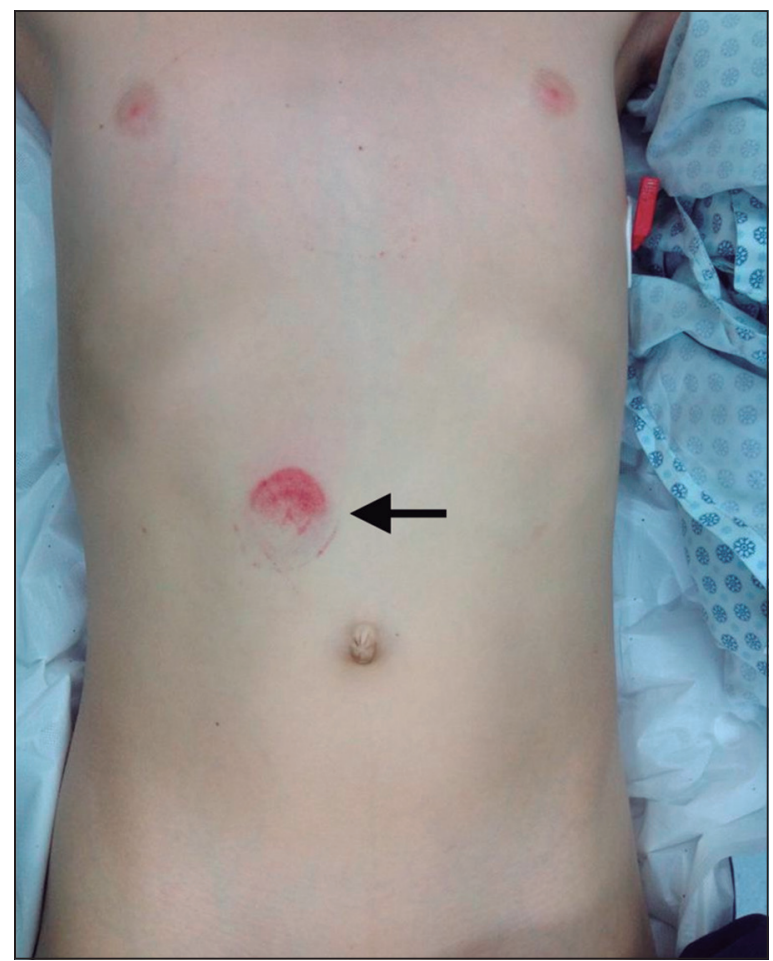

Figura 1. Se observa tatuaje en epigastrio por golpe con manubrio de bicicleta (flecha). Flecha: tatuaje en epigastrio. tercera porción del duodeno, en la cara lateral (figura 3 ). Se realizó duodenorrafia en dos planos en sentido transverso. Se instaló una sonda nasoyeyunal transanastomótica, sonda nasogástrica y drenaje. No se identificaron otras lesiones.

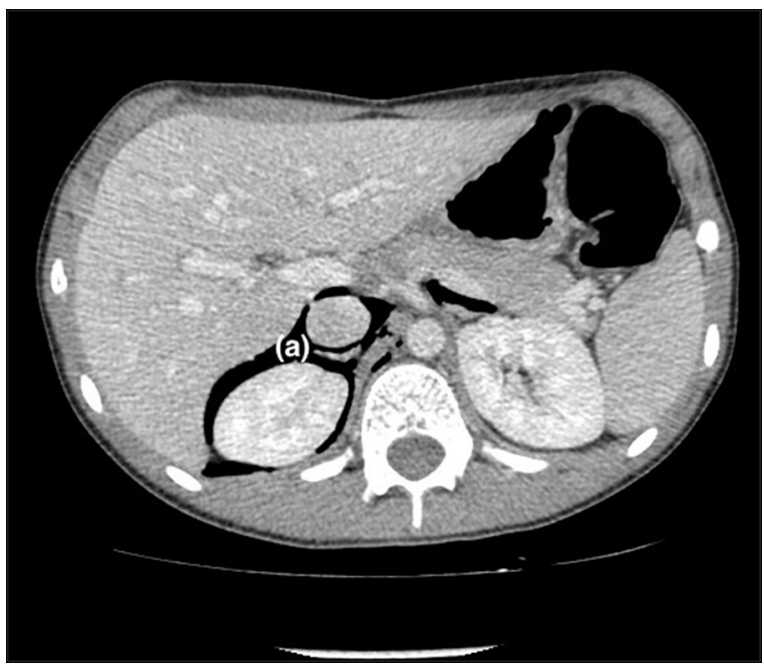

Figura 2. Tomografía computada de abdomen y pelvis con contraste endovenoso. Corte axial. Los hallazgos imageneológicos de neumoretroperitoneo extenso (a) y sangre acumulada en la gotera parietocólica derecha (no visualizado en esta imagen) hacen sospechar una perforación duodenal. (a): neumoretroperitoneo.

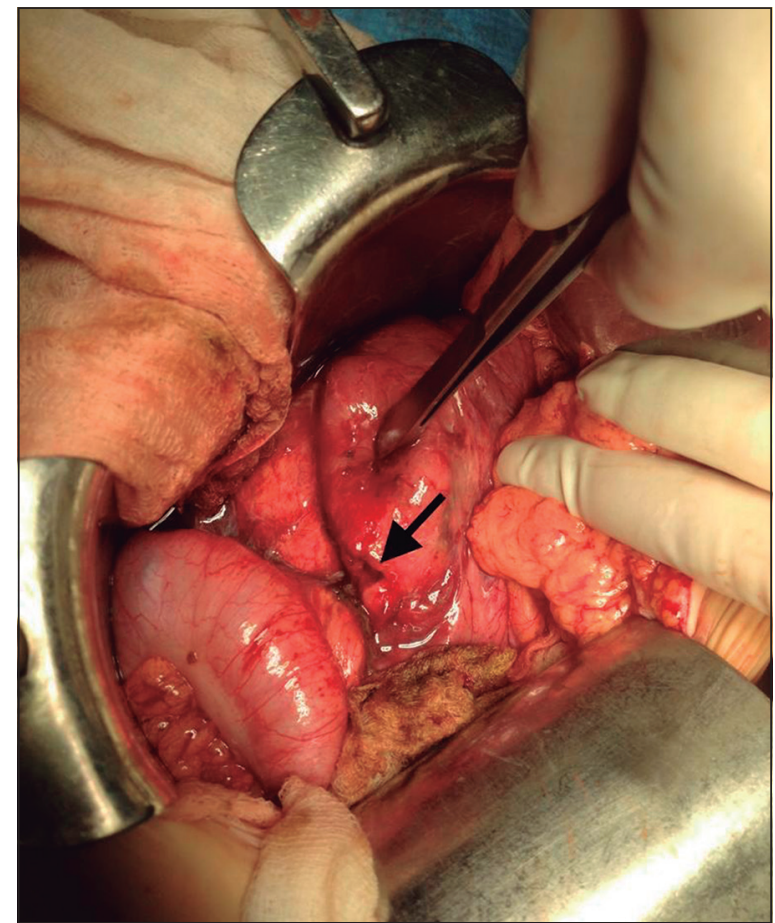

Figura 3. Laparotomía exploradora. Se observa defecto de continuidad en la pared lateral del duodeno, entre su segunda y tercera porción (flecha). Flecha: perforación duodenal. 
El paciente ingresó a la Unidad de Paciente Crítico Pediátrica (UPC) donde permaneció por $24 \mathrm{~h}$. Al segundo día postoperatorio se trasladó a sala. Al quinto día se retiró la sonda nasogástrica y el drenaje, y se reinició alimentación enteral de manera progresiva. El paciente se mantuvo en buenas condiciones siendo dado de alta al noveno día postoperatorio. Se realizó control clínico a los 10 días, 2 meses y 11 meses posteriores al alta, constatando buena evolución.

\section{Caso 2}

Paciente masculino, sano, de 14 años, acudió al servicio de urgencias 60 minutos luego de caída en bicicleta con golpe del manubrio en región toracoabdominal izquierda. Taquicárdico (128 lpm), normotenso y con GCS 15, inmovilizado con collar cervical. Al examen físico se describía pálido, bien hidratado y perfundido. Presentaba escoriaciones superficiales en el hombro derecho y en el hipocondrio y muslo izquierdos. Refería dolor leve a la palpación cervical. Destacaba sensibilidad abdominal difusa, mayor en el hipocondrio izquierdo, asociado a resistencia muscular y signo de Blumberg.

En el servicio de urgencias se realizó reanimación inicial con aporte de volumen en bolos, con lo que normalizó frecuencia cardíaca (83 lpm), y se administró analgesia intravenosa.

Se realizó TC de columna cervical, tórax, abdomen y pelvis. Se descartó lesión de columna y se identificó fractura esplénica de orientación coronal con múltiples laceraciones y signos sugerentes de sangrado arterial activo, sin lesión del hilio; asociado a laceración de la cola del páncreas con presencia de pequeñas colecciones peripancreáticas anteriores y posteriores, líquido libre periesplénico, en la pelvis y en el espacio interasas (figura 4). La lesión esplénica se clasificó como grado
IV-A AAST ${ }^{9}$. Por la presencia de sangrado activo se realizó embolización esplénica por angiografía, la que resultó sin incidentes (figura 5).

Luego de una caída inicial de siete puntos de hematocrito, que requirió transfusión de glóbulos rojos, permaneció estable, monitorizado tres días en UPC. Previo a su traslado a sala se controló con TC de abdomen que mostró resolución de la laceración pancreática con disminución en el tamaño de las colecciones, viabilidad del bazo y reabsorción de hemoperitoneo. Se realimentó sin incidentes y se mantuvo en reposo absoluto por una semana. Reinició la deambulación en forma progresiva y fue dado de alta 48 h más tarde, en buenas condiciones, previo control ecográfico que mostró estabilidad de las lesiones.

Se realizó control clínico y ecografía abdominal a las dos y seis semanas posteriores al accidente, mos-

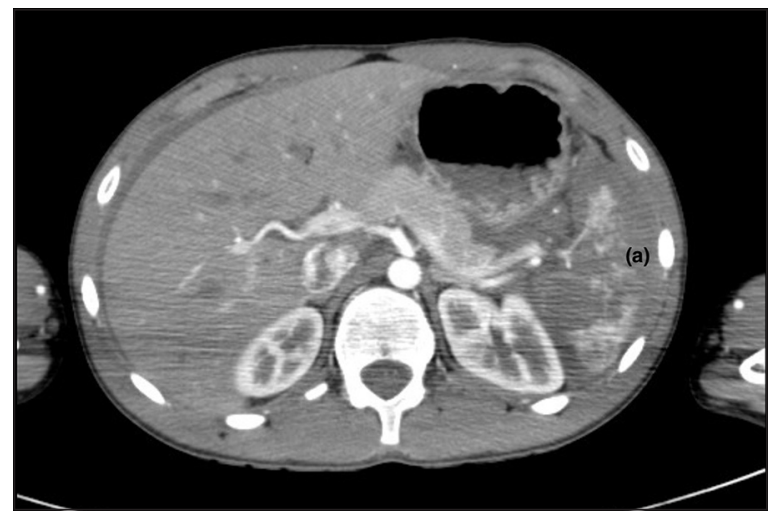

Figura 4. Tomografía computada de abdomen y pelvis con contraste endovenoso. Corte axial. La imagen muestra la impregnación "en parches" del medio de contraste en el bazo (a), hallazgo compatible con estallido esplénico. (a): bazo.

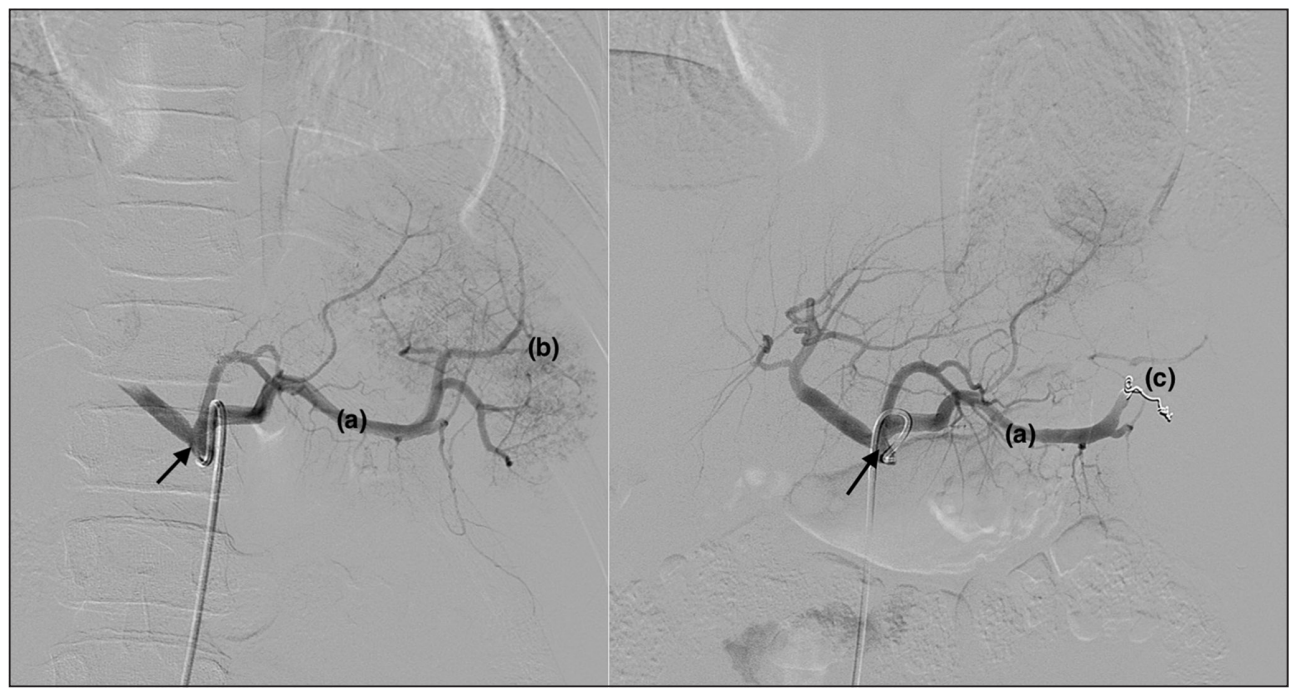

Figura 5. Angiografía. La imagen de la izquierda corresponde al control basal: angiografía con inyección en tronco celíaco (flecha) y arteria esplénica (a) que muestra impregnación sinusoidal del bazo con alteración de la vascularización de tercio medio e inferior (b) concordante con estallido esplénico, sin evidenciar sangrado arterial focal. La imagen de la derecha corresponde a la angiografía post embolización: embolización proximal en hilio esplénico mediante uso de microcoils (c) preservando irrigación de tercio superior esplénico. Flecha: tronco celíaco, (a): arteria esplénica, (b): bazo, (c): microcoils. 
trando un bazo de tamaño e irrigación normal y páncreas sano sin presencia de colecciones ni pseudoquistes. Debido a esto, la edad del paciente y su estabilidad clínica se desestimaron nuevos controles con TC.

\section{Caso 3}

Paciente sano, masculino, de 9 años. Consultó luego de 30 minutos de haber sufrido accidente en bicicleta con impacto del manubrio en hipocondrio derecho. Ingresó estable, normocárdico, hipertenso $(120 / 73)$ y con GCS 15. Al examen físico se describía pálido, con dolor intenso y erosión en hipocondrio derecho, sin signos de irritación peritoneal.

Se solicitó TC de abdomen y pelvis que informó laceración de $40 \mathrm{~mm}$ de longitud en el segmento VI del hígado, asociado a leve hemoperitoneo y engrosamiento parietal del colon a nivel del ángulo hepático, se clasificó como una lesión hepática grado III AAST ${ }^{9}$ (figura 6). En sus exámenes de laboratorio destacaba elevación de las transaminasas (SGOT 293 U/L, SGPT $175 \mathrm{U} / \mathrm{L})$. Se administró volumen en bolo y se manejó el dolor con bomba de analgesia controlada por el paciente, decidiéndose manejo conservador. Ingresó a UPC pediátrica para monitorización y se mantuvo en reposo absoluto hasta que se resolvió el dolor abdominal. Se reinició la alimentación oral en forma progresiva luego de $24 \mathrm{~h}$. Al tercer día se controló TC de abdomen que reveló estabilidad de la lesión. Evolucionó favorablemente y fue dado de alta al sexto día. En control ecográfico a las 2, 8 y 12 semanas posteriores al alta se documentó resolución de la lesión hepática.

\section{Discusión}

Los accidentes en bicicleta son una causa común de trauma en pediatría ${ }^{2-4}$. Sin embargo, en nuestro país no se cuenta con trabajos que describan la frecuencia y características de ellos. Los causados por golpe con el manubrio conforman un subgrupo especial, ya que tienen más riesgo de causar lesiones de órganos intraabdominales. En cambio, los provocados por una caída desde la bicicleta suelen ocasionar lesiones de extremidades, cabeza, cuello y sistema nervioso ${ }^{7}$. El trabajo que reúne mayor número de pacientes fue publicado en 2019, corresponde a una serie retrospectiva de 385 casos de pacientes pediátricos que sufrieron accidentes en bicicleta ${ }^{7}$. El 27,8\% de los accidentes fueron por golpe con el manubrio, de éstos, un 34,6\% sufrió alguna lesión de órganos sólidos y un 9,3\% de vísceras huecas. Del total de dicho grupo, un 41,1\% requirió algún tipo de intervención.

En el presente artículo se describen los casos de tres pacientes expuestos al mismo mecanismo de trauma: el primero con perforación duodenal que requirió manejo quirúrgico; el segundo con una laceración esplénica y

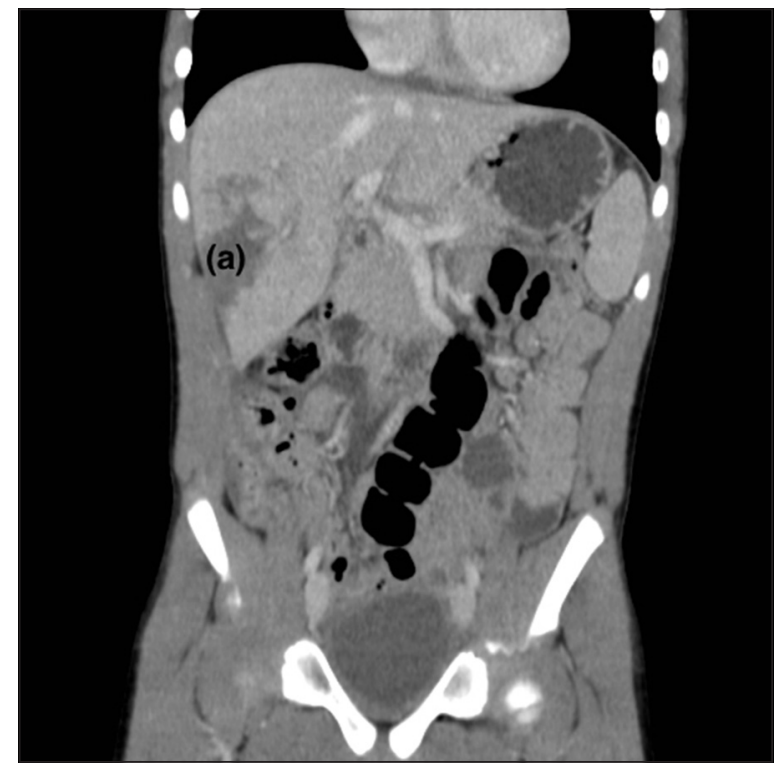

Figura 6. Tomografía computada de abdomen y pelvis con contraste endovenoso. Reconstrucción coronal. En la imagen se observa la laceración del segmento VI hepático (a) de más de 3 cm de profundidad. (a) laceración hepática.

sangramiento activo manejada con angioembolización selectiva y el tercero con una laceración hepática frente a la cual se mantuvo una conducta expectante. Los tres casos contaron con una resolución exitosa, sin requerir reintervenciones, y con un seguimiento que demuestra su evolución favorable. En todos los pacientes se describían lesiones en la piel causadas por el manubrio de bicicleta, las que se correlacionaron espacialmente con las lesiones subyacentes. Es así como la mayoría de las veces existe una correspondencia entre las lesiones de órganos intraabdominales y la ubicación del tatuaje del manubrio en la zona abdominal (figura 1).

El enfrentamiento inicial de cualquier niño con un traumatismo de esta naturaleza debiera seguir los protocolos del programa ATLS, conocidos como el "AB-C" del trauma, que prioriza el tratamiento de las lesiones potencialmente mortales en el corto plazo. Una vez completada la evaluación primaria y secundaria, el examen de elección en niños con trauma abdominal es la TC con contraste, que permitirá identificar las lesiones subyacentes, clasificarlas y definir su manejo ${ }^{10}$.

El diagnóstico de perforación de víscera hueca puede ser difícil e incluso tardío $0^{3,8,13,14}$. Los signos típicos de peritonitis al examen físico, como resistencia muscular o signo de Blumberg, están presentes en alrededor del $50 \%$ de los pacientes ${ }^{13,15}$. El hallazgo de neumoperitoneo o neumoretroperitoneo, como en el Caso 1, está presente sólo en $59 \%$ de los pacientes. En ausencia de él, otros signos importantes de pesquisar en la TC, sugerentes de perforación intestinal son: el 
engrosamiento parietal intestinal, hematoma parietal intestinal y la presencia de líquido libre intraabdominal no explicado por otra causa ${ }^{26}$. En estos casos el manejo debe ser quirúrgico y el Gold standard terapéutico es la laparotomía exploradora ${ }^{13}$. La cirugía tiene una morbilidad de $20 \%$, mortalidad de hasta $5 \%$ y riesgo de obstrucción intestinal a largo plazo de $3 \%{ }^{17}$. En el caso de perforación duodenal, se recomienda instalar una sonda nasoyeyunal para permitir la alimentación enteral precoz, además de una sonda nasogástrica para descompresión, lo que permite disminuir el intervalo al inicio del régimen vía oral ${ }^{13}$. En el paciente del Caso 1 se pudo iniciar el régimen oral de manera progresiva al quinto día posterior al accidente. En este paciente, la contusión epigástrica, pudiera haber comprometido cualquiera de las estructuras anatómicas allí contenidas. En el caso del duodeno, las lesiones tienden a producirse en las porciones retroperitoneales de este órgano, las que al ser fijas, se comprimen contra los elementos de la pared abdominal posterior, como la columna vertebral.

El manejo específico de pacientes pediátricos con lesiones contusas de órganos sólidos, como hígado o bazo, varía según la condición hemodinámica ${ }^{11,18}$. En pacientes estables está indicado el tratamiento médico exclusivo, logrando tasas de éxito cercanas al $90 \%{ }^{10,19}$. El fracaso del manejo conservador ocurre generalmente dentro de las primeras $12 \mathrm{~h}$ y se han identificado factores asociados a un mayor riesgo: shock hipovolémico, hemorragia activa, peritonitis, perforación de víscera hueca, mayor severidad de la lesión y lesión de múltiples órganos ${ }^{10,12,18}$.

Si el paciente se encuentra hemodinámicamente inestable o se anticipa una evolución desfavorable, es necesario un manejo activo ${ }^{3,4}$, que dependerá de las características de la lesión. El abordaje tradicional ha sido la laparotomía exploradora ${ }^{8}$. La utilidad de la laparoscopía se ha planteado en casos seleccionados ${ }^{20}$. La esplenectomía ha sido el tratamiento quirúrgico de elección en las lesiones esplénicas ${ }^{21}$. Sin embargo, se reconoce que los pacientes pediátricos sometidos a esplenectomía por trauma tienen mayor riesgo de complicaciones infecciosas a largo plazo, y es por esto que la preservación del órgano resulta un objetivo especialmente importante en estos pacientes ${ }^{18}$. Por lo anterior, en el paciente del Caso 2, el cual se encontraba sangrando activamente, se decidió realizar angioembolización selectiva con el fin de evitar la esplenectomía. La angioembolización selectiva ha sido descrita como una opción segura y efectiva en pacientes adultos con lesión de órganos sólidos, que se ha extrapolado a pacientes pediátricos ${ }^{19}$. Se plantea como una alternativa para reducir la tasa de fracaso del manejo médico y a la vez evitar el manejo quirúrgico y la esplenectomía con sus posteriores complicaciones ${ }^{21}$. Actualmente no se cuenta con trabajos prospectivos que demuestren el beneficio de la angioembolización en población pediátrica ${ }^{19}$. Esta aproximación debiera considerarse en pacientes que no respondan al manejo médico inicial y en los que se encuentre sangrado activo ${ }^{10,18,22,23}$.

Las pruebas de función hepática orientan hacia la presencia de una lesión hepática en pacientes con examen físico equívoco, o incluso cuando no se sospecha una lesión del hígado, ya que un aumento de las transaminasas por sobre valores normales, es un factor predictor de lesión de dicho órgano ${ }^{3,15}$. La confirmación diagnóstica de un trauma hepático, su clasificación y manejo, deben realizarse con TC con contraste ${ }^{10}$. En el paciente del Caso 3, al ingreso se constató elevación de los niveles de transaminasas, los que fueron concordantes con la lesión hepática identificada en la TC. Al tercer día de hospitalización se repitió el perfil hepático, evidenciándose disminución significativa de las transaminasas, concordante con la estabilidad mostrada en la TC de seguimiento, lo que permitió descartar la presencia de complicaciones.

En pacientes pediátricos con lesiones hepáticas de alto grado, es necesario descartar la presencia de lesión del árbol biliar. Una alternativa es realizar cintigrafía hepática, un método diagnóstico no invasivo, que no requiere anestesia, con alta sensibilidad y especifici$\mathrm{dad}^{24-26}$. En los pacientes donde se ha confirmado una fuga biliar por cintigrafía biliar, o existe una sospecha alta de ésta, el abordaje con colangiopancreatografía retrógrada endoscópica se plantea como opción terapéutica segura y efectiva, con una baja tasa de complicaciones asociadas, que contribuye a evitar el manejo quirúrgico. Su rol es diagnóstico y a la vez terapéutico al ser complementado con inserción de stent o esfinterotomía ${ }^{24-26}$. En el paciente del caso 3, no hubo sospecha de lesión de vía biliar, ya que la laceración se encontraba alejada del árbol biliar. Por ende, se prescindió de los estudios descritos y únicamente se realizó seguimiento con TC descartándose aumento del líquido libre que obligase a tomar una conducta activa.

En el presente trabajo se describen tres casos de pacientes que presentaron trauma abdominal contuso por golpe con manubrio de bicicleta. Aunque comparten el mecanismo, las lesiones observadas fueron diferentes y así también su manejo. La evolución en el corto y largo plazo fue favorable en todos ellos.

En conclusión, el trauma abdominal contuso por manubrio de bicicleta, si bien es poco frecuente, puede llegar a ser potencialmente grave en población pediátrica, pudiendo afectar órganos sólidos y vísceras huecas. El manejo no quirúrgico cada vez es más utilizado, logrando altas tasas de éxito en pacientes estables. Los pacientes inestables, o en los que se sospeche perforación de víscera hueca, requerirán cirugía como primera aproximación. 


\section{Responsabilidades Éticas}

Protección de personas y animales: Los autores declaran que los procedimientos seguidos se conformaron a las normas éticas del comité de experimentación humana responsable y de acuerdo con la Asociación Médica Mundial y la Declaración de Helsinki.

Confidencialidad de los datos: Los autores declaran que han seguido los protocolos de su centro de trabajo sobre la publicación de datos de pacientes.

Derecho a la privacidad y consentimiento informado: Los autores han obtenido el consentimiento informado de los pacientes y/o sujetos referidos en el artículo. Este documento obra en poder del autor de correspondencia.

\section{Conflicto de intereses}

Los autores declaran no tener conflicto de intereses.

\section{Agradecimientos}

Los autores quisieran hacer un especial reconocimiento al Dr. Luis Meneses Quiroz, quien realizó el procedimiento de angioembolización descrito en el caso 2.

\section{Referencias}

1. Instituto Nacional de Estadísticas. Anuario de estadísticas vitales 2017. Santiago, Chile; 2017. Disponible en: https://www.ine.cl/estadisticas/sociales/ demografia-y-vitales/nacimientosmatrimonios-y-defunciones [Accedido el 14 de marzo de 2020].

2. Klimek P, Lutz T, Stranzinger E, Zachariou Z, Kessler U, Berger S. Handlebar injuries in children. Ped Surg Int. 2012;29(3):269-73.

3. Gaines B, Ford H. Abdominal and pelvic trauma in children. Crit Care Med. 2002; 30(Supplement):S416-S423.

4. Hirose T, Ogura H, Kiguchi T, Mizushima Y, Kimbara F, Shimazaki J, et al. The risk of pediatric bicycle handlebar injury compared with non-handlebar injury: a retrospective multicenter study in Osaka, Japan. Scand J Trauma Resusc. Sept 2015;23(66):1-5.

5. Comisión Nacional de Seguridad de Tránsito. Siniestros de tránsito de ocupantes de bicicletas y consecuencias. [Internet]. Santiago, Chile; 2016. Disponible en: https://www.conaset. cl/wp-content/uploads/2017/10/ Bicicletas-2016.pdf. [Accedido el 14 de marzo de 2020].

6. López JJ, Xu ML, Stringel G. Handlebar Injuries in Children: 20 Years of Experience at A Level I Pediatric Trauma Center. J Trauma Care. 2017;3(4):1028.

7. Vandewalle R, Barker S, Raymond J, Brown B, Rouse T. Pediatric Handlebar Injuries. More than meets the abdomen. Pediatr Emerg Care 2019, Jan 21. [Epub ahead of print].

8. Fernández R, Bragagnini P, Álvarez N, Delgado R, García J, Escartín R, et al. Handlebar injury in children: Are we ignoring the signs? J Pediatr Surg. 2015;3(5):215-8.

9. Moore E, Cogbill T, Jurkovich G, Shackford S, Malangoni M, Champion H. Organ Injury Scaling. J Trauma. 1995;38(3):323-4.

10. Notrica D, Linnaus M. Nonoperative Management of Blunt Solid Organ Injury in Pediatric Surgery. Surg Clin N Am. 2016;97(1):1-20.

11. Notrica D, Eubanks J, Tuggle D, Maxson R, Letton R, García N, et al. Nonoperative management of blunt liver and spleen injury in children. J Trauma. 2015;79(4):683-93.

12. Holmes J, Wiebe D, Tataria M, Mattix K, Mooney D, Scaife E, et al. The Failure of Nonoperative Management in Pediatric Solid Organ Injury: A Multi-institutional Experience. J Trauma. 2005;59(6):130913.

13. Bruny J, Bensard D. Hollow viscous injury in the pediatric patient. Semin Pediatr Surg. 2004;13(2):112-8.

14. Lam J. Lesson of the week: Delayed presentation of handlebar injuries in children. BMJ. 2001;322(7297):1288-9.

15. Holmes J, Sokolove P, Brant W, et al. Identification of Children with Intraabdominal Injuries after Blunt Trauma. Ann Emerg Med. 2002;39(5):500-9.

16. Mirvis SE, Gens DR, Shanmuganathan K. Rupture of the bowel after blunt abdominal trauma: diagnosis with CT. Am J Roentgenol. 1992;159:1217-21.

17. Feliz A, Shultz B, McKenna C, Gaines B. Diagnostic and therapeutic laparoscopy in pediatric abdominal trauma. J Pediatr Surg. 2006;41(1):72-7.

18. Naess P, Gaarder C, Dormagen J. Nonoperative management of pediatric splenic injury with angiographic embolization. J Pediatr Surg. 2005;40(11):e63-e64.

19. Swendiman RA, Goldshore MA, Fenton SJ, et al. Defining the Role of Angioembolization in Pediatric Isolated Blunt Solid Organ Injury. J Pediatr Surg. May 2019 [en prensa] [Accedido el 14 de marzo de 2020]. doi: https://doi. org/10.1016/j.jpedsurg.2019.04.036.

20. Tharakan SJ, Kim AG, Collins JL, et al. Laparoscopy in pediatric abdominal trauma: a 13 year experience. Eur J Pediatr Surg. 2016;26(5):443-44.

21. Rialon K, Englum B, Gulack B, et al. Comparative effectiveness of treatment strategies for severe splenic trauma in the pediatric population. Am J Surg. 2015;212(4):786-93.

22. Gates R, Price M, Cameron D, et al. Non-operative management of solid organ injuries in children: An American Pediatric Surgical Association Outcomes and Evidence Based Practice Committee systematic review. J Pediatr Surg. 2019;54(8):1519-26.

23. Drexel S, Azarow K, Jafri M. Abdominal Trauma Evaluation for the Pediatric Surgeon. Surg Clin N Am. 2017;97(1):59 74.

24. Notrica D. Pediatric blunt abdominal trauma. Curr Opin Crit Care. 2015;21(6):531-7.

25. Kulaylat A, Stokes A, Engbrecht B, McIntyre J, Rzucidlo S, Cilley R. Traumatic bile leaks from blunt liver injury in children: A multidisciplinary and minimally invasive approach to management. J Pediatr Surg. 2014;49(3):424-7.

26. Garvey E, Haakinson D, McOmber M, Notrica D. Role of ERCP in pediatric blunt abdominal trauma: A case series at a level one pediatric trauma center. J Pediatr Surg. 2015;50(2):335-8. 